\title{
Numerical simulation of non-isothermal absorption in a liquid film moving over a cooled horizontal surface under the action of a gas flow
}

\author{
Maria V. Bartashevich ${ }^{1, *}$ \\ ${ }^{1}$ Kutateladze Institute of Thermophysics, 630090 Novosibirsk, Russia
}

\begin{abstract}
Mathematical model of absorption on a horizontal film of lithium bromide water solution flowing on a cooled wall under the action of shear stress is numerically investigated in this paper. The shear stress on the film surface is set by the motion of co-current or counter-current surrounding saturated water vapor flow. It is known that the external shear stress is caused by the motion of surrounding saturated water vapor intensificate the process of non-isothermal absorption in comparison with the fixed-vapor regime. Our calculations have shown that at low values of heat flux the film temperature and vapor concentration in the solution downstream increases due to absorption.
\end{abstract}

\section{Problem statement}

The necessity of heat and mass transfer intensification in the absorption chillers is caused by the limitations towards their size and consumption of materials. The use of thin liquid films just solves these problems. Our numerical modelling of the conjugated heat and mass transfer in absorption chillers focuses on the film flow process on cooling wall. The liquid film moving on a cooled horizontal wall under the action of co-current or counter-current vapor flow have been investigated. The liquid film of lithium-bromide water solution has been considered. The lithium bromide-water solution widely used in absorption refrigeration systems due to its effectivity especially for low heat temperatures [1]. The non-isothermal absorption takes place in the various energy devices [2,3]. The investigation of thin liquid film flowing down a vertical wall in the roll-wave regime in presence of heat and mass transfer through the free surface was presented in [4,5]. The absorption and desorption processes under the conditions, corresponding to the operating modes of thermal transformers are described in [6]. The analytical solution to the problem of conjugate heat and mass transfer in a laminar falling liquid film with a linear velocity profile is presented in [7]. The results of the numerical simulation of absorption and their comparison with the experimental data are presented in [8]. The results of experimental studies concerning the development of critical phenomena and structure formation in the process of boiling in falling films and during liquid cavitation were presented in [9]. Also, in [10,11] it was

\footnotetext{
Corresponding author: bartashevichmv@gmail.com
} 
shown that free convection in the gas significantly affects the desorption (adsorption) of the aqueous solution of the salt.

We assume, that the lithium bromide-water solution liquid film flowing under the action of the external tangential stress on the film surface. The external shear stress is caused by the motion of surrounding saturated water vapor. It is assumed that the shear stress applied to the interface will allow intensification of the process of non-isothermal absorption in comparison with the fixed-vapor regime. We consider the two-dimensional stationary flow of a laminar liquid film of thickness $h$ over a horizontal plate. The surface of liquid is in contact with moving vapor. The shear stress on the film surface is $\tau$. Absorption is considered in the framework of ordinary assumptions [2,3]. The thermalphysical properties of solution are assumed constant.

Let us introduce the Cartesian coordinate system with $O x$ axis in the direction of the flow and $O y$ axis directed along the normal to the plate. The velocity profile in the film takes form

$$
u=\frac{y \tau}{\mu}
$$

The process of heat and mass transfer at film absorption is described by the equations of heat conductivity and diffusion:

$$
\begin{aligned}
& u \frac{\partial T}{\partial x}=a \frac{\partial^{2} T}{\partial y^{2}}, \\
& u \frac{\partial C}{\partial x}=D \frac{\partial^{2} C}{\partial y^{2}},
\end{aligned}
$$

$a$ is thermal diffusivity, $\mathrm{m}^{2} / \mathrm{s} ; D$ is diffusion coefficient, $\mathrm{m}^{2} / \mathrm{s}$. The boundary conditions at the inlet at $x=0$ are:

$$
T=T_{i}, C=C_{i} .
$$

Similarly to [1], the equilibrium of solution-vapor system occurs on the interface at $y=h$ :

$$
C_{d}=f\left(T_{d}\right), \lambda \frac{\partial T}{\partial y}=\chi \frac{\partial C}{\partial y} .
$$

where $\chi=L \rho D /\left(1-C_{i}\right), L$ is latent heat of phase transformation, $\mathrm{J} / \mathrm{kg} ; \rho$ is solution density, $\mathrm{kg} / \mathrm{m}^{3} ; \lambda$ is heat conductivity, $\mathrm{W} /(\mathrm{mK})$. This equilibrium condition joins equilibrium temperature $T_{d}$ with concentration $C_{d}$. We set on the wall at $y=0$ :

$$
\left.\lambda \frac{\partial T}{\partial y}\right|_{y=0}=q_{w},\left.\frac{\partial C}{\partial y}\right|_{y=0}=0
$$

where $q_{w}$ is heat flux on the wall. The conditions of absorption are $T_{i}<T_{s}$ and $C_{i}<C_{s}$. Here, $C_{s}=f\left(T_{i}\right)$ is equilibrium concentration, corresponding to initial temperature of solution $T_{i} ; T_{s}$ is equilibrium temperature corresponding to initial concentration $C_{i}$ ( 
$\left.C_{i}=f\left(T_{s}\right)\right)$, similarly to [1]. Then we turn to dimensionless variables $\xi=x / l$, $l=\tau h^{3} /(\mu a), \eta=y / h, \theta=\left(T-T_{i}\right) /\left(T_{s}-T_{i}\right)$.

\section{Numerical calculations}

Numerical calculations were carried out by the finite difference method. The results of calculations of absorption on a film moving under the action of a tangential stress over a horizontal cooled wall are given in Fig. $1-$ Fig. 3. The calculations were performed at $T_{i}=$ $20{ }^{\circ} \mathrm{C}, T_{s}=46{ }^{\circ} \mathrm{C}, C_{i}=0.4, C_{s}=0.56, \bar{q}_{w}$ is a dimensionless heat flux. Fig. 1 shows the calculated distributions of the dimensionless temperature along the film surface for different heat fluxes on the wall. Corresponding concentration distributions vs. longitudinal coordinate are shown in Fig. 2. Fig. 3 shows the calculated distributions of the dimensionless concentration at different distances from the inlet.

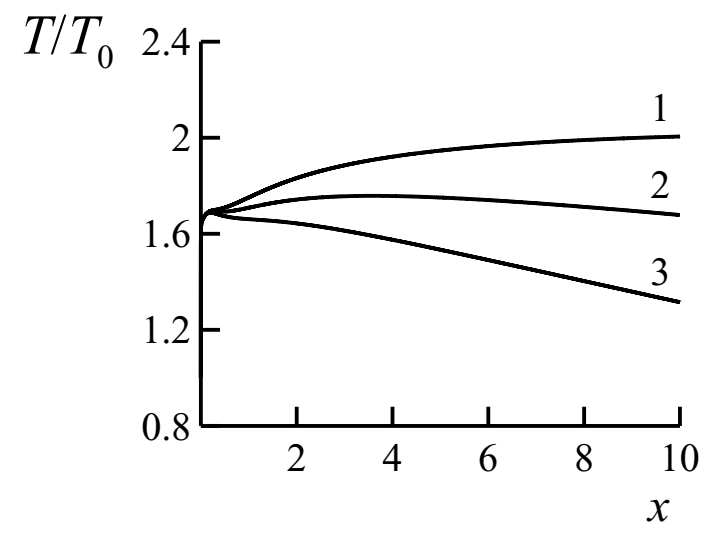

Fig. 1. Profiles of dimensionless temperature of the film surface at different heat fluxes: $1-\bar{q}_{w}=0.01 ; 2-\bar{q}_{w}=0.1 ; 3-\bar{q}_{w}=0.2$.

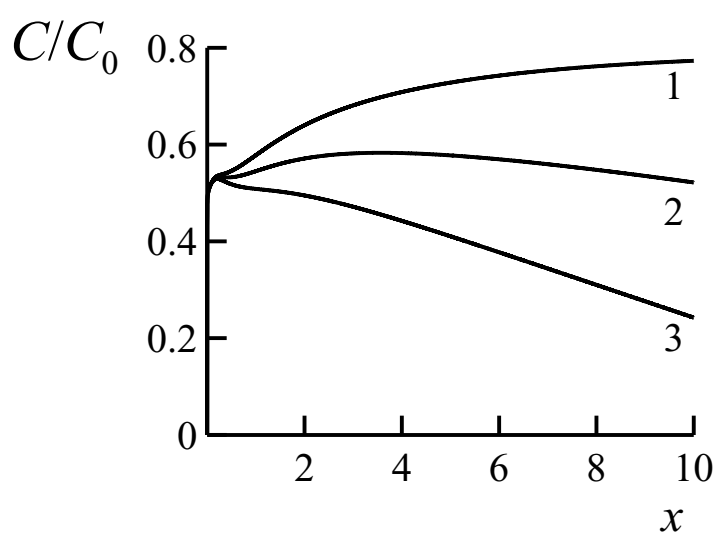

Fig. 2. Profiles of dimensionless concentration on the film surface at different heat fluxes: $1-\bar{q}_{w}=0.01 ; 2-\bar{q}_{w}=0.1 ; 3-\bar{q}_{w}=0.2$. 


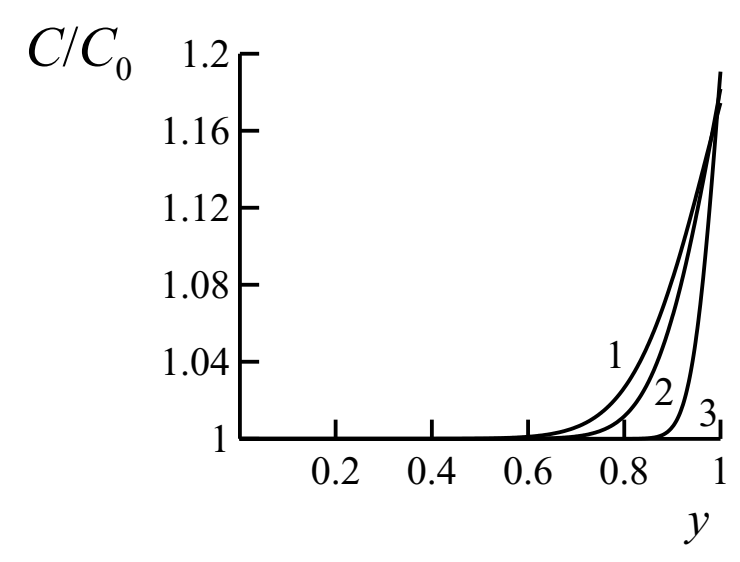

Fig. 3. Profiles of dimensionless concentration in different cross-sections of the film, $\bar{q}_{w}=$ $0.01 .1-\xi=0.1 ; 2-\xi=0.5 ; 3-\xi=0.8$.

\section{Conclusions}

Mathematical model of absorption on a horizontal film of lithium bromide water solution flowing on a cooled wall under the action of shear stress for the given constant heat flux is numerically investigated in this paper. The shear stress on the film surface is set by the motion of co-current or counter-current surrounding saturated water vapor flow. It is shown that at low values of heat flux the film temperature and vapor concentration in the solution downstream increases due to absorption.

The study was funded by FASO Russia.

\section{References}

1. J. Yin, L. Shi, M.-S. Zhu, L.-Z. Han, Appl. Energy 67 (2000)

2. V.E. Nakoryakov, N.I. Grigorieva, Non-Isothermal Absorption in Thermal Transformers (Nauka, Novosibirsk, 2010)

3. V.E. Nakoryakov, N.I. Grigoryeva, M.V. Bartashevich, Int. J. Heat Mass Transfer 54 (21-22) (2011)

4. V.E. Nakoryakov, V.V. Ostapenko, M.V.Bartashevich, Int. J. Heat Mass Transfer 55 (23-24) (2012)

5. V.E. Nakoryakov, V.V. Ostapenko, M.V.Bartashevich, Int. J. Heat Mass Transfer 89 (2015)

6. M. Mittermaier, F. Ziegler, Int. J. Refrig. 59 (1) (2015)

7. M. Mortazavi, S. Moghaddam, Int. J. Refrig. 66 (2016)

8. E. Garcia-Rivera, J. Castro, J. Farnos, A. Oliva, Int. J. Therm. Sci. 109 (2016)

9. A. N. Pavlenko, V. P. Koverda, V. N. Skokov, A. V. Reshetnikov, A. V. Vinogradov, A. S. Surtaev, J. Eng. Thermophys. 18 (1) (2009)

10. S.Ya. Misyura, Sci. Rep. 7, 14759 (2017)

11. S.Ya. Misyura, Int. J. Heat Mass Transfer 116 (2017) 